
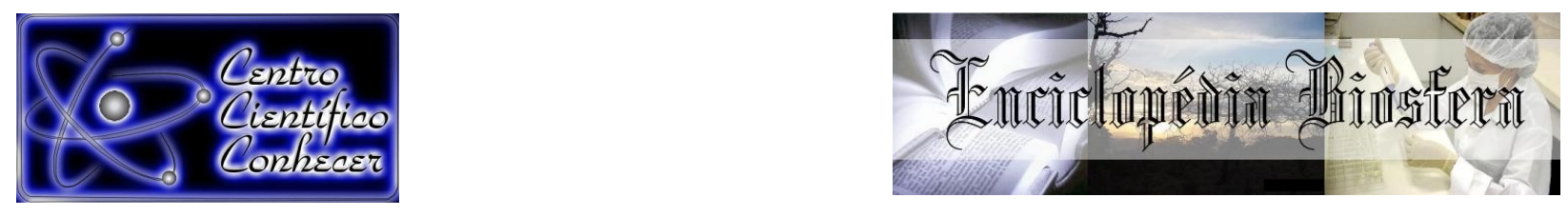

\title{
APROVEITAMENTO DE RESÍDUO DE MADEIRA DE Hymenaea courbaril PARA PRODUÇÃO DE PAINÉIS AGLOMERADOS CONVENCIONAIS EM COMPARAÇÃO COM PAINÉIS DE Pinus oocarpa
}

Karollyna Macedo Carvalho"; _José Benedito Guimarães Junior²; Bárbara Maria Ribeiro Guimarães ${ }^{3}$; Rafael Farinassi Mendes²; Lourival Marin Mendes ${ }^{4}$

1. Graduanda em Engenharia Florestal - Regional Jataí - Universidade Federal de Goiás

2. Prof. Dr. Ciência e Tecnologia da Madeira - Universidade Federal de Lavras jbguimaraesjr@hotmail.com

3. Pós-Graduando em Engenharia de Biomateriais - Departamento de Engenharia Florestal - Universidade Federal de Lavras

4. Professor Dr. em Ciência Florestais - Departamento de Engenharia Florestal Universidade Federal de Lavras

Recebido em: 08/04/2017 - Aprovado em: 10/06/2017 - Publicado em: 20/06/2017 DOI: 10.18677/EnciBio 2017A12

\section{RESUMO}

O acúmulo de resíduos de serraria, principalmente serragem pode comprometer o meio ambiente causando impactos com danos de difícil reparação, necessitando assim serem corretamente manejados. Objetivou-se neste trabalho avaliar o potencial técnico de utilização de resíduos de serraria de Hymenaea courbaril para produção de painéis aglomerados convencionais, comparando com painéis de Pinus oocarpa. Foram produzidos 3 painéis por tratamento, sendo que os mesmos apresentaram dimensões de $20,0 \times 20,0 \times 1,5 \mathrm{~cm}$ e densidade nominal de 0,60 $\mathrm{g} / \mathrm{cm}^{3}$. Aplicou-se adesivo uréia-formaldeído na proporção de $9 \%$ com base no seu teor de sólidos. O ciclo de prensagem utilizado foi de temperatura de $160^{\circ} \mathrm{C}$, pressão de 4,0MPa e tempo de 15 minutos. Com base nos resultados pode-se verificar que o resíduo de jatobá atende todas as normativas, tendo potencial técnico de ser utilizado na produção de chapas aglomeradas.

PALAVRAS- CHAVE: Jatobá, serragem, serraria.

\author{
WOOD WASTE USE OF Hymenaea courbaril FOR THE PRODUCTION OF \\ CONVENTIONAL PARTICLEBOARD IN COMPARISON WITH PANELS OF PinUS \\ oocarpa
}

\begin{abstract}
The accumulation of sawmill waste, mainly sawdust can compromise the environment causing impacts with damages of difficult repair, needing to be properly managed. The objective of this work was to evaluate the technical potential of using sawmills from Hymenaea courbaril for the production of conventional agglomerated panels, comparing with panels of Pinus oocarpa. Three panels were produced per treatment, being that they had dimensions of $20.0 \times 20.0 \times 1.5 \mathrm{~cm}$ and nominal
\end{abstract}


density of $0.60 \mathrm{~g} / \mathrm{cm}^{3}$. Urea-formaldehyde adhesive was applied at a ratio of $9 \%$ based on its solids content. The press cycle used was of temperature of $160^{\circ} \mathrm{C}$, pressure of $4,0 \mathrm{MPa}$ and time of 15 minutes. Based on the results it can be verified that the jatobá residue meets all the regulations, having the technical potential to be used in the production of particleboard.

KEYWORDS: Jatobá, sawdust, sawmill.

\section{INTRODUÇÃO}

O setor madeireiro no Brasil apresenta grande produção e consequentemente uma alta geração de resíduos, se caracterizando por ser uma indústria com baixo rendimento. Se os mesmos forem despejados no ambiente, podem acarretar problemas de poluição, principalmente durante a incineração sem um prévio controle ambiental. MELO (2012) destaca que a solução para essa problemática passa pela adoção de políticas adequadas, de forma que seja possível o aproveitamento do potencial apresentado por estas. Este ainda salienta que isso será imprescindível no futuro, uma vez que a escassez da matéria-prima florestal fará com que empresários do setor busquem alternativas para manter as atividades.

Os resíduos da madeira podem ser encontrados na forma de serragem, maravalha e cavacos. Dentre estes, destaca-se a serragem, que é o resíduo que tem entre $0,5 \mathrm{~mm}$ a $2,5 \mathrm{~mm}$ e é obtido nos processos de corte e usinagem das peças de madeira (desdobro primário e secundário). Estes materiais podem causar impactos ambientais, pois tem características de ser combustível e alta biodegradabilidade, ocasionando problemas quando estiverem estocados de maneira inadequada, próximos a aglomerações urbanas (WINDER, 2015).

Esses resíduos, quando corretamente identificados e quantificados, têm grande potencial de serem reutilizados, sendo uma alternativa, no sentido de obter produtos de maior valor agregado ou ser incorporado em outros processos produtivos, tais como produção de polpa celulósica, painéis reconstituídos, geração de energia, produção de etanol, dentre outros (FARIA et al., 2016).

Os painéis aglomerados são chapas provenientes de partículas de madeira ou materiais lignocelulósicos, coladas entre si com adesivo sintético (MOSLEMI, 1974). De acordo com GUIMARÃES JUNIOR et al. (2016) e MENDES et al. (2012) os painéis aglomerados convencionais apresentam, por conceito, a possibilidade para utilização de matérias primas de menor qualidade, como resíduos florestais ou agroindustriais na produção.

PIERRE et al. (2014) relatam que a utilização de resíduos do setor florestal, tais como os gerados pela colheita, desrama, madeiras de qualidade inferior e pelo setor indústria de serrarias e marcenarias, já são amplamente utilizados para produção de painéis aglomerados em outros países, principalmente europeus.

A espécie Hymenaea courbaril, é uma espécie de arbórea e ocorre tanto em solos de alta como de média fertilidade. É conhecida vulgarmente como jatobá, jutaí, jutaí-açu, jutaí-bravo, jutaí-grande, jataí-peba, jataí-uba, jataí-uva, jataíba, jataúba, jatioba, jatiuba ou jupati, copal. É encontrada nos estados da Bahia, Minas Gerais e São Paulo. Apresenta copa ampla e tronco reto. A madeira é considerada resistente, tendo como principais utilizações a construção civil, postes, estacas, móveis e laminados (CIPRIANO et al., 2014).

Neste sentido, o objetivo deste trabalho foi avaliar o potencial técnico de utilização de serragem de madeira de Hymenaea coubaril para produção de painéis ENCICLOPÉDIA BIOSFERA, Centro Científico Conhecer - Goiânia, v.14 n.25; p.128 
aglomerados, comparando-o com painéis produzidos com madeira de Pinus oocarpa.

\section{MATERIAL E MÉTODOS}

O experimento foi realizado em novembro de 2016 na Universidade Federal de Goiás - Regional Jataí, no laboratório de Tecnologia dos Produtos Florestais. Foram confeccionados três painéis com madeira de Pinus e três com serragem de Jatobá. A serragem utilizada como matéria prima desta pesquisa foi coletada em serraria localizada em Jataí-GO. A espécie escolhida foi a Hymenaea courbaril (Jatobá), por ser amplamente utilizada na região para confecção de móveis. A madeira de Pinus oocarpa foi obtida na Universidade Federal de Lavras, de plantio experimental, com idade de 18 meses. Esse material foi cedido já na forma de partículas. Optou-se pela madeira de pinus por ser a mais empregada no Brasil para produção de aglomerados, servindo como bom parâmetro para se inferir sobre o potencial de utilização dos resíduos de serraria.

Esses materiais foram peneirados, sendo utilizadas para produção dos painéis aquelas partículas que ficaram retidas entre as peneiras de 10 e 30 mesh. Posteriormente as partículas foram secas em estufa com circulação forçada de ar até umidade na base seca de $3 \%$. O adesivo utilizado foi a ureia-formaldeído. Aplicou-se $9 \%$ em relação à massa seca de partícula. $O$ adesivo apresentou as seguintes características: teor de sólidos de $53,80 \%$, pH de 8,65, viscosidade de 430 cP e tempo de gelatinização de 50 segundos.

$\mathrm{Na}$ sequência, as partículas foram misturadas manualmente com adesivo uréia-formaldeído, não sendo aplicada emulsão de parafina. O colchão de partículas foi pré-prensado, com pressão nominal de $0,5 \mathrm{MPa}$, por cinco minutos, em temperatura ambiente. Posteriormente, o painel passou pelo ciclo de prensagem, com temperatura de $160^{\circ} \mathrm{C}$ e pressão específica de $4,0 \mathrm{MPa}$, por um período de 15 minutos, obtendo-se, assim, os painéis homogêneos.

Foram produzidos três painéis para cada um dos dois tratamentos. As chapas foram confeccionadas com dimensões nominais de $220 \mathrm{~mm} \times 220 \mathrm{~mm} \times 15 \mathrm{~mm}$ e $0,60 \mathrm{~g} / \mathrm{cm}^{3}$ de densidade nominal. As seguintes propriedades físico-mecânicas foram determinadas conforme a Tabela 1.

TABELA 1. Ensaios físicos e mecânicos realizados nos painéis aglomerados.

\begin{tabular}{ccc}
\hline Ensaio & $\begin{array}{c}\text { Número de } \\
\text { amostras por } \\
\text { painel }\end{array}$ & Norma \\
\hline $\begin{array}{c}\text { Flexão } \\
\text { estática }\end{array}$ & 4 & Deutsche Institut für Normung - DIN, 1982 \\
\hline $\begin{array}{c}\text { Tração } \\
\text { perpendicular }\end{array}$ & 5 & American Society for Testing and Materials - ASTM, 1999 \\
\hline $\begin{array}{c}\text { Absorção de } \\
\text { água e }\end{array}$ & & \\
$\begin{array}{c}\text { Inchamento } \\
\text { em }\end{array}$ & & American Society for Testing and Materials - ASTM, 1999 \\
espessura & 5 & MALONEY (1993) \\
\hline $\begin{array}{c}\text { Razão de } \\
\text { compactação }\end{array}$ & 5 & \\
\hline
\end{tabular}


Utilizou-se o delineamento inteiramente casualizado, com três repetições, em que os tratamentos foram considerados os dois materiais utilizados na produção dos painéis aglomerados. $\mathrm{O}$ tratamento com painéis produzidos com Pinus oocarpa foi considerado controle. Utilizou-se o teste de Tuckey a $5 \%$ de significância.

\section{RESULTADOS E DISCUSSÃO}

$\mathrm{Na}$ Figura 1 pode-se observar os valores médios de densidade aparente e razão de compactação para os painéis aglomerados. Nota-se que para densidade aparente os painéis não apresentaram diferenças significativas, apresentando valor médio de $0,54 \mathrm{~g} / \mathrm{cm}^{3}$. Dessa forma, os painéis são classificados como de baixa densidade, de acordo com a norma CS 236-66 (COMMERCIAL STANDARD - CS, 1968). As densidades aparentes médias dos painéis se diferiram da densidade nominal pré-estabelecida de $0,60 \mathrm{~g} / \mathrm{cm}^{3}$. Fato que pode ser justificado devido a perda de partículas durante a formação do colchão, assim como também pode estar relacionado ao retorno em espessura dos painéis após a retirada da prensa quente e acondicionamento, com consequente aumento do volume e redução da densidade nominal inicial.

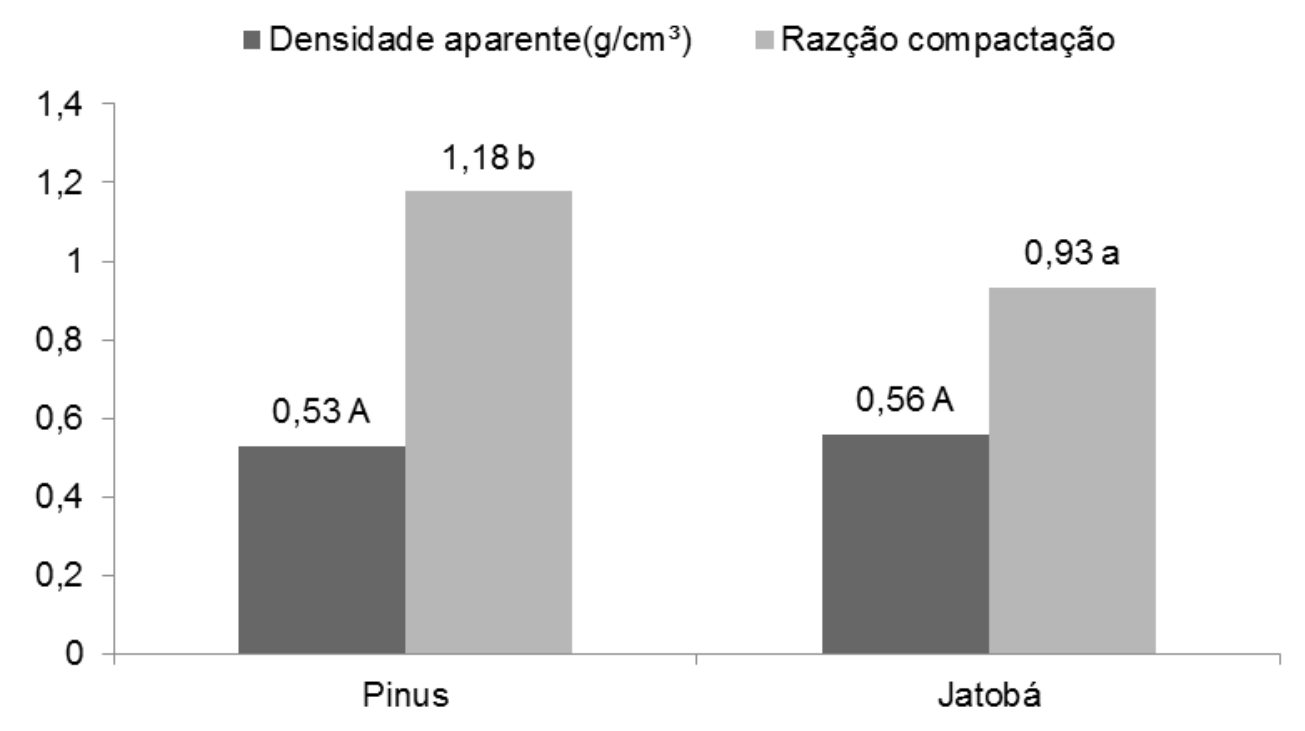

FIGURA 1. Densidade aparente e razão de compactação em painéis aglomerados de Pinus oocarpa e Hymenaea courbaril.

Para razão de compactação notou-se que os painéis produzidos com resíduo de jatobá apresentaram menores valores. Tais resultados se devem a alta densidade do material $\left(0,65 \mathrm{~g} \mathrm{~cm}^{-}{ }^{3}\right)$ em comparação com a madeira de pinus $(0,40 \mathrm{~g}$ $\left(\mathrm{cm}^{3}{ }^{3}\right.$ ). Isso porque a razão de compactação é determinada pela relação entre a densidade do painel e a densidade do material lignocelulósico que o gerou. 
Na Figura 2 estão apresentados os resultados de absorção de água após duas e 24 horas de imersão para os painéis aglomerados. Pode-se observar que não houve efeito significativo das matérias-primas utilizadas nestas propriedades. Estes valores ficaram próximos aos observados por BIANCHE et al. (2012) estudando madeiras de Eucalypytus urophylla, Schizolobium amazonicum e Sida spp. para produção de painéis aglomerados, encontraram valores médios de absorção após duas e 24 horas: $17,41 \%$ a $84,45 \%$ e $57,62 \%$ a $107,38 \%$, respectivamente.

Os valores observados por IWAKIRI et al. (2012) foram menores aos observados nesta pesquisa, avaliando painéis aglomerados produzidos com resíduos de serrarias de nove espécies de madeiras tropicais, obtendo valores de absorção de água entre duas e 24 horas variando de 6,77\% a 17,62\%, e 18,43 a $34,70 \%$, respectivamente.

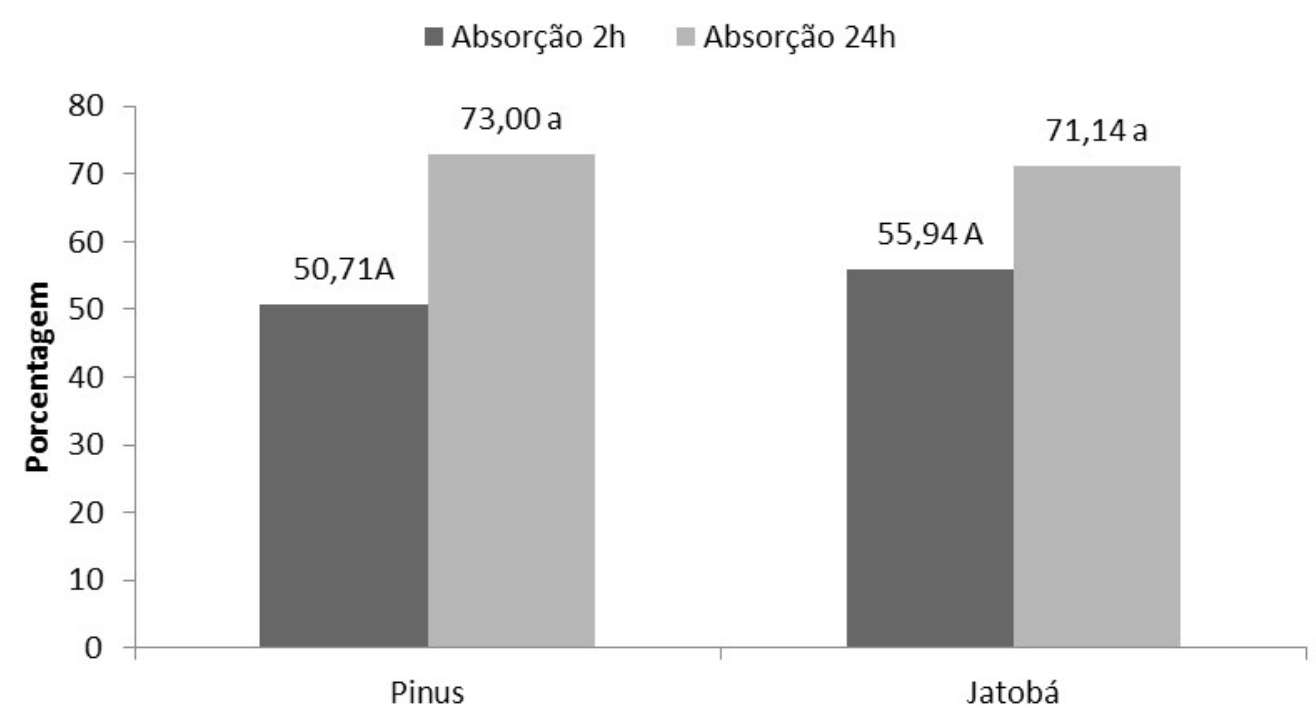

FIGURA 2. Absorção de água após duas e 24 horas de imersão de painéis aglomerados de Pinus oocarpa e Hymenaea courbaril.

Os valores médios obtidos para as propriedades de inchamento em espessura após duas e 24 horas de imersão em água estão apresentados na Figura 2. Os painéis produzidos com resíduo de serraria da madeira de Jatobá e de Pinus não apresentaram diferenças significativas entre as médias. Os resultados obtidos nesta pesquisa estão próximos ao observados por MENDES et al. (2014) trabalhando com painéis aglomerados de resíduo da laminação de clones de Eucalyptus urophylla, encontraram valores para inchamento em espessura após duas e 24 horas entre 13,3 a $28,8 \%$ e 29,9 e $40,8 \%$, respectivamente.

A norma CS 236-66 (COMMERCIAL STANDARD, 1968) exige para comercialização de painéis valores de inchamento em espessura, após 24 horas de imersão em água, de no máximo $30 \%$ para painéis de baixa densidade. Nesse caso, ambos painéis atenderam a normatização. 


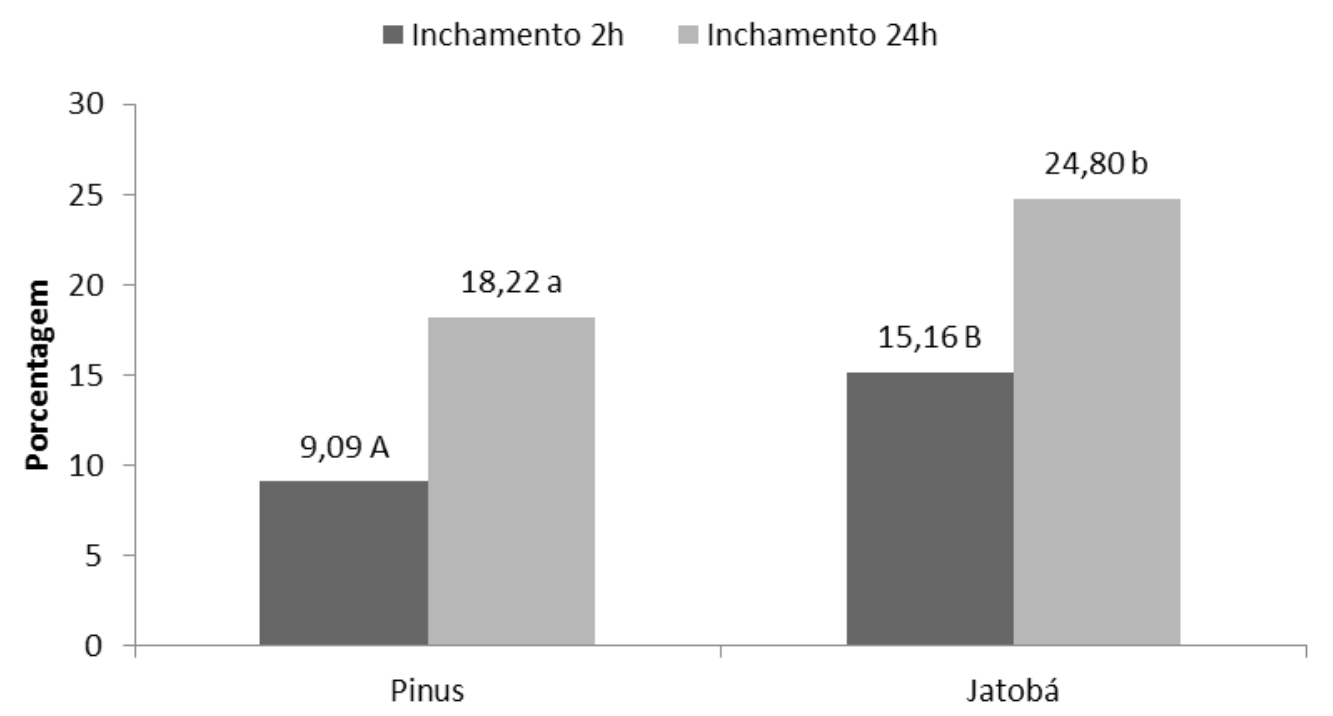

FIGURA 3. Inchamento em espessura após duas e 24 horas de imersão de painéis aglomerados de Pinus oocarpa e Hymenaea courbaril.

$\mathrm{Na}$ Tabela 2 são apresentados os valores médios dos módulos de ruptura (MOR) e elasticidade (MOE) a flexão estática e tração perpendicular a superfície para os tratamentos propostos.

TABELA 2. Propriedades mecânicas em painéis aglomerados de Pinus oocarpa e Hymenaea courbaril.

\begin{tabular}{cccc}
\hline Tratamentos & MOE $(\mathrm{MPa})$ & MOR $(\mathrm{MPa})$ & $\begin{array}{c}\text { Tração perpendicular } \\
(\mathrm{MPa})\end{array}$ \\
\hline Pinus & $3085,60 \mathrm{~B}$ & $14,00 \mathrm{~A}$ & $0,3658 \mathrm{~A}$ \\
Jatobá & $1116,86 \mathrm{~A}$ & $14,97 \mathrm{~A}$ & $0,3947 \mathrm{~A}$ \\
\hline CV $(\%)$ & 19,23 & 28,12 & 17,76 \\
\hline
\end{tabular}

Os resultados mostraram que o MOE nos painéis de Pinus $(3085,60 \mathrm{MPa})$ foi superior ao encontrado para o Jatobá $(1116,86)$. Já para o MOR não foram constatadas diferenças estatisticamente significativas entre as espécies estudadas, sendo valor médio dos tratamentos de $14,44 \mathrm{MPa}$.

Esses resultados ficaram próximos aos observados por RACHTANAPUN et al. (2012) trabalhando com painéis de resíduos de casca de café, utilizando $11 \%$ de ureia-formaldeído, encontrando MOR e MOE médios de 8,54 e $1116 \mathrm{MPa}$, respectivamente. Também o resultado da flexão estática desta pesquisa ficou próximo do observado por BIANCHE et al. (2012), que produziram painéis a partir de partículas de vassoura (Sida sp.), e encontraram valores de 13,13 e 1154,1 MPa para MOR e MOE, respectivamente. 
De acordo com a norma de comercialização CS 236-66 (COMMERCIAL STANDARD, 1968), os painéis aglomerados produzidos com adesivo ureia formaldeído e de baixa densidade, devem apresentar, no mínimo, 1.052 MPa para MOE e 5,6MPa para MOR realizados na flexão estática. Dessa forma ambos os tratamentos se adequaram a normatização.

Para tração perpendicular verificou-se que não houve diferença significativa para os materiais estudados. O valor médio para essa propriedade foi de $0,3803 \mathrm{MPa}$. Estes valores estão dentro do intervalo encontrado por SCATOLINO et al. (2013) avaliando diferentes percentagens de sabugo de milho em associação com madeira de Pinus oocarpa (0; 25; 50; 75 e 100\%) em painéis aglomerados consolidados com $8 \%$ de uréia-formaldeído (1,13 a 0,23 MPa).

A norma de comercialização CS 236-66 estabelece valor mínimo de 0,1400MPa para tração perpendicular nos painéis de baixa densidade e produzidos com adesivo ureia formaldeído. Neste sentido, ambos os tratamentos atenderam as exigências normativas.

\section{CONCLUSÃO}

Com base nos resultados obtidos nesta pesquisa, pode-se concluir que os painéis produzidos com resíduo de serraria da madeira de Hymenaea courbaril apresentaram todas as propriedades físicas e mecânicas iguais as da madeira de Pinus oocarpa (com exceção do MOE), que é a principal fonte de matéria-prima utilizada pela indústria de painéis aglomerados.

Os painéis aglomerados produzidos com resíduo de serraria da madeira de Jatobá apresentaram todas as propriedades estudadas dentro das exigências normativas, mostrando a viabilidade técnica de produção dos mesmos com a qualidade exigida pelo mercado.

\section{REFERÊNCIAS}

BIANCHE, J. J.; CARNEIRO, A. C. O.; VITAL, B. R.; PEREIRA, F. A.; SANTOS, R. C.; SORATTO, D. N. Propriedades de painéis aglomerados fabricados com partículas de eucalipto (Eucalyptus urophylla), paricá (Schizolobium amazonicum) e vassoura (Sida spp.). Cerne, v.8, n.4, p.623-630, 2012. DOI: 10.1590/01047760.201420021289.

CIPRIANO J.; MARTINS, L.; DEUS, M. S. M.; PERON, A. N. O gênero Hymenaea e suas espécies mais importantes do ponto de vista econômico e medicinal para o brasil. Caderno de Pesquisa. Série Biologia, v. 26, n.2 , p.41-51, 2014. DOI: 10.17058/cp.v26i2.5248.

COMMERCIAL STANDARD. Mat formed wood particleboard.CS 236-66. In: Book of Commercial Standards. Wallingford, 1968.

FARIA, W. S.; PROTÁSIO, T.; TRUGILHO, P. F.; PEREIRA, B. L. C.; CARNEIRO, A. D. C. O., ANDRADE, C. R.; JUNIOR, J. B. G. Transformação dos resíduos 
lignocelulósicos da cafeicultura em pellets para geração de energia térmica. Coffee Science, Lavras, v. 11, n. 1, p. 137 - 147, jan./mar. 2016. DOI: 1050-6258-1-PB $\% 20(6)$.

GUIMARÃES JUNIOR, J. B., XAVIER, M. M., SANTOS, T. S., PROTÁSIO, T., MENDES, R. F., MENDES, L. M. Inclusão de resíduo da cultura de sorgo em painéis aglomerados de eucalipto. Pesquisa Florestal Brasileira, 36(88), 435-442. 2016. DOI: $10.4336 / 2016 . p f b .36 .88 .1036$.

IWAKIRI, S.; VIANEZ, B. F.; WEBER, C.; TRIANOSKI, R.; ALMEIDA, V. C. Avaliação das propriedades de painéis aglomerados produzidos com resíduos de serrarias de nove espécies de madeiras tropicais da Amazônia. Acta Amazônica, Manaus, v. 42, n.1, p. 59-64, 2012. DOI: 10.1590/S0044-59672012000100007

MELO, R.R. Avaliação de variáveis tecnológicas na produção de painéis LVL confeccionados com paricá (Schizolobium amazonicum Huber ex. Ducke). Tese de Doutorado em Ciências Florestais, Publicação PPGEFL.TD - 026/2012, Departamento de Engenharia Florestal, Universidade de Brasília, Brasília, DF, 164p

MENDES, R. F.; MENDES, L. M.; MENDONÇA, L. L.; GUIMARÃES JR., J. B.; MORI, F. A. Qualidade de painéis aglomerados homogêneos produzidos com a madeira de clones de Eucalyptus urophylla. Cerne, Lavras, v. 20, n. 2, p. 1-10, jan./mar. 2014. DOI: 10.1590/01047760.201420021273.

MENDES, R. F.; MENDES, L. M.; GUIMARÃES JUNIOR, J. B.; SANTOS, R. C.; CÉSAR. A. A. S. Efeito da associação de bagaço de cana, do tipo e do teor de adesivo na produção de painéis aglomerados. Ciência Florestal, Santa Maria, v. 22, n. 1, p. 161-170, jan/1mar, 2012. DOI: 10.5902/198050985088

MOSLEMI, A.A. Particleboard: Materials. Illinois: Southern Illinois University Press, 1974. v.1. $244 p$

PIERRE, F. C.; BALLARIN, A. W.; PALMA, L. Caracterização física de painéis aglomerados de Eucalyptus grandis com adição de resíduos industriais madeireiros. Cerne, Lavras, v. 20, n. 2, p. 321-328, abr./jun. 2014. DOI: 10.1590/01047760.201420021289.

RACHTANAPUN, P.; SATTAYARAK, T.; KETSAMAK, N. Correlation of density and properties of particleboard from coffee waste with urea-formaldehyde and polymeric methylene diphenyl diisocyanates. Journal of Composite Materials, v.15, n.46, p.1839- 1850, 2012. DOI: 10.1177/0021998311426624.

SCATOLINO, M. V.; SILVA, D. W.; MENDES, R. F.; MENDES, L. M. Maize cob utilization in particleboards production. Ciência e Agrotecnologia, Lavras, v. 37, n. 4, 2013. DOI: $10.1590 / \mathrm{S} 1413-70542013000400006$.

WILDNER, M. V. "Reaproveitamento de resíduos da indústria moveleira para aplicação em novos produtos de mobiliário". 2015. Monografia (Graduação em 
Design) - Centro Universitário UNIVATES, Lajeado, jun. 2015. Disponível em: <http://hdl.handle.net/10737/867>. 